\title{
Layer-oriented multigrid wavefront reconstruction algorithms for multiconjugate adaptive optics
}

Luc Gilles, Brent L. Ellerbroek, Curtis R. Vogel

Luc Gilles, Brent L. Ellerbroek, Curtis R. Vogel, "Layer-oriented multigrid wavefront reconstruction algorithms for multiconjugate adaptive optics," Proc. SPIE 4839, Adaptive Optical System Technologies II, (7 February 2003); doi: 10.1117/12.459347

SPIE Event: Astronomical Telescopes and Instrumentation, 2002, Waikoloa, Hawai'i, United States 


\title{
Layer-Oriented Multigrid Wavefront Reconstruction Algorithms for Multi-Conjugate Adaptive Optics
}

\author{
L.Gilles $^{a}$, B.L.Ellerbroek ${ }^{b}$ and C.R.Vogel ${ }^{c}$ \\ ${ }^{a}$ Michigan Technological University, Dept. of Electrical and Computer Engineering, \\ Houghton, MI 49931-1295 \\ ${ }^{b}$ Gemini Observatory, 670 N. A'Ohoku Place, Hilo, HI 96720 \\ ${ }^{c}$ Montana State University, Dept. of Mathematical Sciences, \\ Bozeman, MT 59717-2400
}

\begin{abstract}
Multi-conjugate adaptive optics (MCAO) systems with $10^{4}-10^{5}$ degrees of freedom have been proposed for future giant telescopes. Using standard matrix methods to compute, optimize, and implement wavefront control algorithms for these systems is impractical, since the number of calculations required to compute and apply the reconstruction matrix scales respectively with the cube and the square of the number of AO degrees of freedom. In this paper, we develop an iterative sparse matrix implementation of minimum variance wavefront reconstruction for telescope diameters up to $32 \mathrm{~m}$ with more than $10^{4}$ actuators. The basic approach is the preconditioned conjugate gradient method, using a multigrid preconditioner incorporating a layer-oriented (block) symmetric Gauss-Seidel iterative smoothing operator. We present open-loop numerical simulation results to illustrate algorithm convergence.
\end{abstract}

Keywords: Multi-Conjugate Adaptive Optics, Wavefront Reconstruction, Multigrid

\section{INTRODUCTION}

Conventional Adaptive Optics (AO) has now successfully been implemented on ground-based imaging systems, ${ }^{1-3}$ and there is a growing interest in developing, what will be the next step in imaging through volume turbulence, wide field imaging using Multi-Conjugate Adaptive Optics (MCAO). ${ }^{4-8}$ Using multiple guide stars and multiple deformable mirrors (DM's), MCAO aims at breaking the small field of view (FoV) barrier inherent in conventional AO and the conical anisoplanatism inherent when using laser guide stars (LGS's). Such systems represent a huge leap in complexity from single mirror systems, and the task of creating an optimal scheme to control them is far from being trivial.

Wavefront reconstructors estimate a set of DM commands from wavefront sensor (WFS) slope measurements. Real-time wavefront reconstructors employ either a vector-matrix-multiply (VMM) or an iterative algorithm to compute the commands. Direct evaluation of the control matrix becomes increasingly difficult as the number of actuators $n$ increases, since the processing time to compute the matrix and apply it to sensor data with a VMM scales approximately respectively as $n^{3}$ and $n^{2}$ (i.e. $D^{6}$ and $D^{4}$ where $D$ is the aperture diameter) when standard matrix multiplication and inversion methods are employed. The control matrix may be computed in a few hours to a few days on a fast workstation for an AO system with $n=10^{3}$, but MCAO systems on extremely large telescopes (ELT's) may have $n=10^{4}-10^{5}$. Relying upon improvements in processor speed and parallel computing to cope with a factor of $10^{3}-10^{6}$ increase in computation requirements is impractical, and the development of new iterative algorithms and advanced numerical and mathematical techniques is highly desirable to help develop and evaluate conceptual designs for MCAO on ELT's.

For conventional AO systems with a single DM and WFS, significant improvements in computational efficiency are possible by exploiting the sparse structure of the deformable mirror influence matrices and atmospheric turbulence inverse covariance matrices appearing in these calculations. Sparse matrix techniques

Send correspondence to L.Gilles 
exploiting the sparse structure of the deformable mirror influence matrices have reduced the overall cost of evaluating and applying the classical least-squares wavefront control matrix defined as the pseudoinverse of the DM-to-WFS influence matrix, to $\mathcal{O}\left(n^{3 / 2}\right)$. For MCAO systems employing multiple DM's and WFS's, the cost of computing and applying the least-squares reconstructor is a much larger constant multiple of $n^{3 / 2}$, and the reconstructor suffers from WFS noise amplification for weakly sensed modes. Some sort of filtering of the pseudoinverse like a truncated singular value decomposition (SVD) appears necessary, but this cancels out the computational advantages of the sparse techniques. Optimum results can be achieved using a minimum variance estimator that includes a regularization term based upon the second-order statistics of atmospheric turbulence and WFS measurement noise. ${ }^{6,7}$

Sparse matrix techniques have successfully been applied to the MCAO minimum variance reconstructor, and have reduced the overall computations to $\mathcal{O}\left(n^{3 / 2}\right)$ using a small adjustment of the turbulence statistics so that the regularization term appearing in the optimal estimator is sparse. ${ }^{9}$ For extreme adaptive optics (ExAO) systems (ultra-high-contrast AO system using a single very high-order DM and WFS), a minimum variance reconstructor based on an iterative multigrid (multiresolution) method scaling as $n \log n$ has also been shown to be very effective, ${ }^{10}$ and a Fourier transform recontructor scaling as $n \log n$ has also been proposed. ${ }^{11}$ This last method directly inverts the phase-to-WFS influence matrix in the Fourier domain using an extension method, but suffers from noise amplification.

In this paper, we address the mathematical and computational issues for MCAO systems (in particular the dramatic amount of fill-in occuring in the Cholesky factorizations ${ }^{9}$ due to the long-range cross-coupling between layers), and report on new ideas for fast and efficient iterative algorithmic approaches based on combined sparse matrix and multigrid techniques that scale in overall computational complexity and real time implementation as $\mathcal{O}\left(n^{3 / 2}\right)$ with a smaller constant multiply. In addition, storage requirements are much smaller. The basic approach is the conjugate gradient method with a multigrid preconditioner to speed up convergence, and a layer-oriented (block) symmetric Gauss-Seidel smoother inspired by the recent layer-oriented control algorithms for MCAO. ${ }^{12-15}$

\section{PROBLEM STATEMENT}

A schematic MCAO system is illustrated in Fig.1. In this system, the WFS's are optically conjugate to the telescope's primary mirror and the DM's to different altitudes $z_{m}^{a}$ in the atmosphere. The WFS's collect light from several natural and/or laser guide stars located within the FoV at an altitude $z_{g} \leq \infty$. Turbulent layers are located at distinct ranges $z_{l}^{\varphi}$. Fig.1 illustrates the LGS case with $z_{g}$ finite.

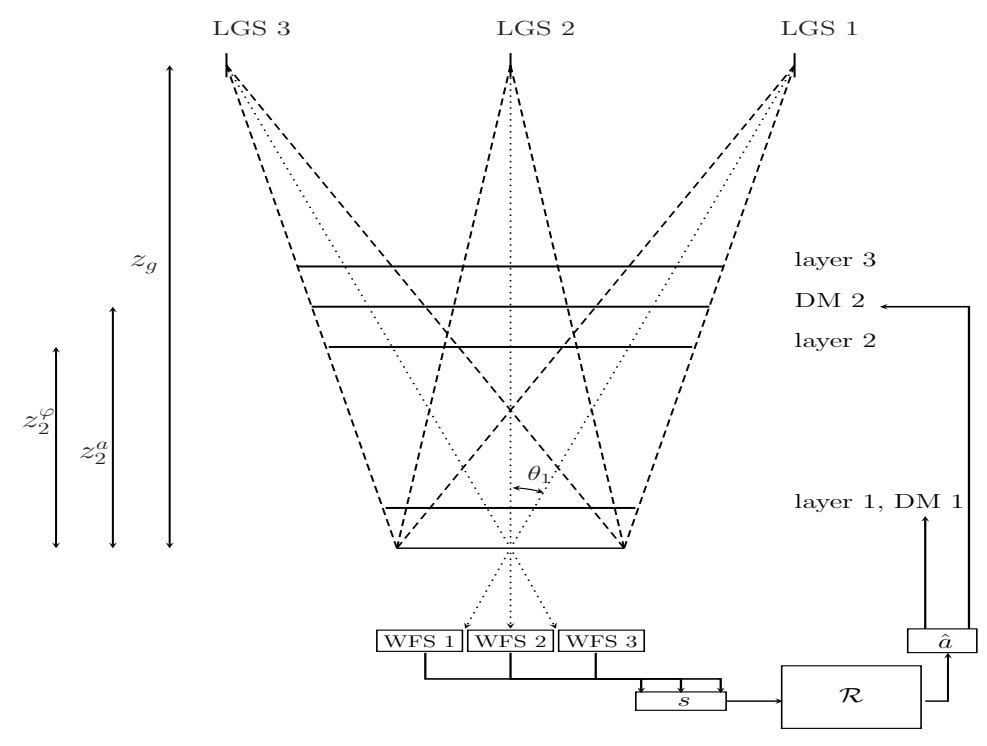

Figure 1. Schematic MCAO system. 
Minimum variance wavefront reconstructors are now favored for MCAO. ${ }^{16}$ For the most general case of either a natural guide star (NGS) or a laser guide star (LGS) MCAO system with $M$ DM's, $H$ high-order WFS's, $T$ full-aperture tip/tilt WFS's (applies only to the LGS case) and $L$ atmospheric layers, with $N \geq L \geq M$, where $N=H+T$ is the total number of WFS's, these reconstructors are block matrix systems of the form

$$
\hat{a}=\mathcal{R}\left(\begin{array}{c}
s \\
\tilde{s}
\end{array}\right), \quad \mathcal{R}=\left(\mathcal{R}_{m n}\right)_{\substack{1 \leq m \leq M \\
1 \leq n \leq N}},
$$

where $\hat{a}, s$ and $\tilde{s}$ are concatanations of respectively $M, H$ and $T$ subvectors corresponding to the estimated actuator commands $a_{m}(1 \leq m \leq M)$, high-order $s_{h}(1 \leq h \leq H)$ and full-aperture tip/tilt $\tilde{s}_{t}(1 \leq t \leq T)$ WFS measurements. The beam print at layer $l$ in direction $\theta_{n}$ together with the metapupil are shown in Fig.2.

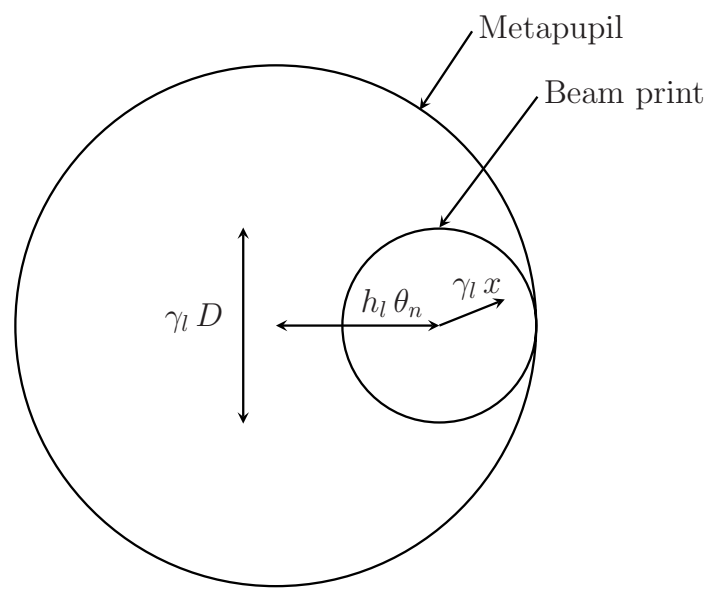

Figure 2. Metapupil and beam print. $x$ is the pupil-plane coordinate, $\gamma_{l}=1-h_{l} / z_{g}$ where $h_{l}$ and $z_{g}$ are respectively layer $l$ and LGS altitudes, with $z_{g}=\infty$ for the NGS case.

For open-loop wavefront sensing, the measurement vector is modeled as

$$
\left(\begin{array}{c}
s \\
\tilde{s}
\end{array}\right)=\mathcal{G} \varphi+\left(\begin{array}{c}
\eta \\
\tilde{\eta}
\end{array}\right), \quad \mathcal{G} \stackrel{\text { def }}{=}\left(\begin{array}{c}
(I-P) G \\
\widetilde{G}
\end{array}\right)
$$

where $\varphi, \eta$ and $\tilde{\eta}$ are concatenations of respectively $L, H$ and $T$ subvectors corresponding to the true turbulence profile $\varphi_{l}(1 \leq l \leq L)$, the high-order $\eta_{h}(1 \leq h \leq H)$ and tip-tilt $\eta_{t}(1 \leq t \leq T)$ WFS measurement noise, assumed to have zero mean and finite second-order statistics. $P$ is a block diagonal orthogonal tip-tilt projector (tilt removal operator) with respect to the high-order WFS's inverse covariance matrix $C^{-1}=\left\langle\eta \eta^{\mathrm{T}}\right\rangle^{-1}$ (applies only to the LGS case):

$$
\begin{gathered}
P=\operatorname{Diag}\left(P_{h}\right)_{1 \leq h \leq H}, \\
P_{h}=K_{h}\left(K_{h}^{\mathrm{T}} C_{h}^{-1} K_{h}\right)^{-1} K_{h}^{\mathrm{T}} C_{h}^{-1}, \\
P_{h}^{\mathrm{T}}=C_{h}^{-1} P_{h} C_{h} \quad \text { (orthogonality) },
\end{gathered}
$$

where $C_{h}=\left\langle\eta_{h} \eta_{h}^{T}\right\rangle$ is the diagonal noise covariance matrix of high-order WFS $h$, and $K_{h}$ is a rank 2 constant matrix (corresponding to the tip and tilt modes) whose number of rows is equal to the number of sensor $h$ grid points. $\left(K_{h}^{\mathrm{T}} C_{h}^{-1} K_{h}\right)$ is thus a $2 \times 2$ matrix and $P$ is a low rank matrix $(\operatorname{rank}(P)=2 H)$ compared to the total number of high-order sensor grid points (number of rows of $P$ ). The phase-to-WFS influence matrices $G$ and $\widetilde{G}$ have the block matrix representation:

$$
G=\left(\Gamma_{h} H_{h l}^{\varphi}\right)_{\substack{1 \leq h \leq H \\ 1 \leq l \leq L}} \quad, \quad \widetilde{G}=\left(\widetilde{\Gamma}_{t} \widetilde{H}_{t l}^{\varphi}\right)_{\substack{1 \leq t \leq T \\ 1 \leq l \leq L}} .
$$


In our computations $\Gamma_{h}$ is a second-order finite difference (FD) approximation of the gradient operator whose stencil depends on the high-order WFS's geometry, and $\widetilde{\Gamma}_{t}$ is the full-aperture tip-tilt projector for NGS WFS $t$ $\left(\operatorname{rank}\left(\widetilde{\Gamma}_{t}\right)=2\right)$. For NGS MCAO systems, $P$ and $\widetilde{G}$ are null matrices, and $\tilde{s}, \tilde{\eta}$ null vectors. To compute phase values at arbitrary coordinates intersecting rays from direction $\theta_{n}$, i.e. at $\left(\gamma_{l} x+\theta_{n}^{x} h_{l}, \gamma_{l} y+\theta_{n}^{y} h_{l}\right)$ for layer $l$, we use the interpolation formula

$$
u^{\mathrm{I}}(x, y)=\sum_{i} e_{i}(x, y) u_{i},
$$

where $e_{i}(x, y)$ is the influence function associated with grid point $i$. We are using here bilinear splines. ${ }^{17}$ Using a column or row grid point ordering scheme, equation (2.5) can be rewritten in matrix form

$$
u^{\mathrm{I}}=H u .
$$

where $u^{\mathrm{I}}$ indicates interpolated quantity and the columns of the interpolation matrix $H$ are the influence functions $e_{i}(x, y)$. In our context, we denote by

$$
\varphi_{n l}^{\mathrm{I}}=H_{n l}^{\varphi} \varphi_{l},
$$

the interpolated phase vector at layer $l$ in direction $\theta_{n}$. For laser guide stars at altitude $z_{g}, \gamma_{l}=\left(1-h_{l} / z_{g}\right)$ (beam reduction if $\left.z_{g}<\infty\right)$. Note also that $H_{n 1}^{\varphi}=I$ since layer 1 is at ground level and therefore no shift or beam reduction is needed. Figure 3 is a schematic illustrating a typical geometry using influence functions.

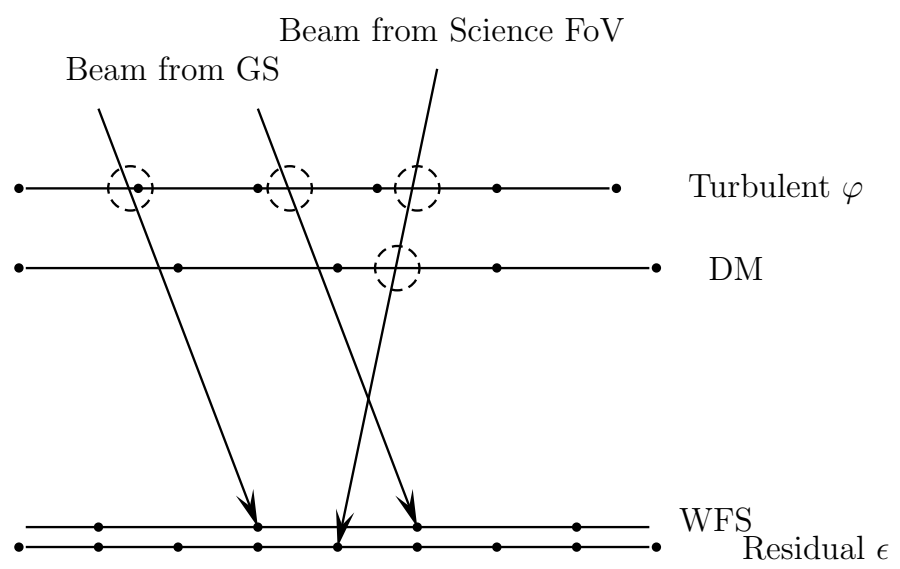

Figure 3. Typical geometry using influence functions.

The minimum variance reconstructor minimizes $\left\langle\|\epsilon\|^{2}\right\rangle$ where $\langle\cdot\rangle$ indicates averaging over turbulence and noise statistics, and $\|\epsilon\|^{2}$ is the normalized aperture-plane, piston removed mean square wavefront error (mse) (overall measure of performance) remaining after the estimated commands $\hat{a}$ have been applied to the DM's, averaged over $\bar{N}$ observation directions within the FoV $(\bar{N} \geq N)$ with weights $\left\{\omega_{\bar{n}}\right\}_{1 \leq \bar{n} \leq \bar{N}}$ :

$$
\|\epsilon\|^{2} \stackrel{\text { def }}{=} \epsilon^{\mathrm{T}} \mathcal{W} \epsilon /\left(H^{\varphi} \varphi\right)^{\mathrm{T}} \mathcal{W}\left(H^{\varphi} \varphi\right)
$$

where $\epsilon$ is a concatanation of $\bar{N}$ subvectors $\epsilon_{\bar{n}}$ corresponding to the different observation directions. The weighting matrix $\mathcal{W}=\operatorname{Diag}\left(\omega_{\bar{n}} W\right)_{1 \leq \bar{n} \leq \bar{N}}$ accounts for aperture edge effects to obtain a more accurate residual wavefront variance, and must define a positive definite metric found by setting the norm squared of any apertureplane vector $u,\|u\|_{W}^{2}=u^{\mathrm{T}} W u$, equal to norm squared of the corresponding continuous function as given by (2.5):

$$
\|u\|_{W}^{2}=\int d x d y \mathcal{A}(x, y)\left[u^{\mathrm{I}}(x, y)-\int d x^{\prime} d y^{\prime} \mathcal{A}\left(x^{\prime}, y^{\prime}\right) u^{\mathrm{I}}\left(x^{\prime}, y^{\prime}\right)\right]^{2},
$$


$\mathcal{A}(x, y)$ being the normalized aperture function $\left(\int \mathcal{A}(x, y) d x d y=1\right)$. The entries of $W$ are then easily identified as

$$
\begin{gathered}
W=W_{0}-v_{1} v_{1}^{\mathrm{T}},(\text { sparse }+ \text { low rank }) \\
\left(W_{0}\right)_{i j}=\int d x d y \mathcal{A}(x, y) e_{i}(x, y) e_{j}(x, y), \\
\left(v_{1}\right)_{i}=\int d x d y \mathcal{A}(x, y) e_{i}(x, y)
\end{gathered}
$$

The overall weighting matrix $\mathcal{W}$ may thus be decomposed as a sparse plus low-rank matrix:

$$
\begin{gathered}
\mathcal{W}=\mathcal{W}_{0}-\mathcal{W}_{1} \mathcal{W}_{1}^{\mathrm{T}}, \\
\mathcal{W}_{0}=\operatorname{Diag}\left(\omega_{\bar{n}} W_{0}\right)_{1 \leq \bar{n} \leq \bar{N}}, \\
\mathcal{W}_{1}=\operatorname{Diag}\left(\omega_{\bar{n}}^{1 / 2} v_{1}\right)_{1 \leq \bar{n} \leq \bar{N}}
\end{gathered}
$$

The pupil-plane residual piston-removed wavefront $\epsilon_{\bar{n}}$ in direction $\bar{n}$ is given by

$$
\begin{gathered}
\epsilon_{\bar{n}}=\left(H_{\bar{n} l}^{\varphi}\right)_{1 \leq l \leq L} \varphi-\left(H_{\bar{n} m}^{a}\right)_{1 \leq m \leq M} \hat{a} \\
=\sum_{l=1}^{L} \varphi \frac{\mathrm{I}}{\bar{n} l}-\sum_{m=1}^{M} \hat{a}_{\bar{n} m}^{\mathrm{I}},
\end{gathered}
$$

where $H_{\bar{n} m}^{a}$ is the interpolation matrix for DM $m$ in direction $\theta_{\bar{n}}$, whose columns are the actuator influence functions $e_{i}^{a}(x)$ associated with each grid point $i$ of DM $m$ at altitude $h_{m}$, evaluated at $\gamma_{m} x+\theta_{\bar{n}} h_{m}$. The actuator influence function $e_{i}^{a}(x)$ models the response of the $i$ th actuator of DM $m$ to a unit command.

The minimum variance reconstructor can be decomposed as

$$
\mathcal{R}=F E .
$$

The estimated DM commands $\hat{a}$ can then be computed in two steps as follows:

$$
\hat{\varphi}=E\left(\begin{array}{c}
s \\
\tilde{s}
\end{array}\right), \quad \hat{a}=F \hat{\varphi}, \quad E=\left(E_{l n}\right)_{\substack{1 \leq l \leq L \\
1 \leq n \leq N}}, \quad F=\left(F_{m l}\right)_{\substack{1 \leq m \leq M \\
1 \leq l \leq L}} .
$$

The estimation matrix $E$ applied to slope measurements provides an estimate $\hat{\varphi}$ of the true turbulence profile $\varphi$, and the fitting matrix $F$ applied to $\hat{\varphi}$ provides a best fit of the actuator commands $\hat{a} .^{7}$

\section{MINIMUM VARIANCE ESTIMATION MATRIX}

The minimum variance estimation (MVE) matrix $E$ is formally given by

$$
\begin{gathered}
E=B^{-1} \mathcal{G}^{\mathrm{T}} \mathcal{C}^{-1}, \quad \mathcal{C}^{-1}=\operatorname{Diag}\left(C^{-1}, \widetilde{C}^{-1}\right), \\
B=\mathcal{G}^{\mathrm{T}} \mathcal{C}^{-1} \mathcal{G}+\mathcal{L}^{-1}, \quad \mathcal{L}^{-1}=\operatorname{Diag}\left(\mathcal{L}_{l}^{-1}\right)_{1 \leq l \leq L},
\end{gathered}
$$

where $C, \widetilde{C}$ are the diagonal sensor noise covariance matrices corresponding respectively to the high-order and full-aperture tip/tilt WFS's, and $\mathcal{L}$ is the block diagonal atmospheric covariance matrix (different turbulent layers are statistically independent). The first step of the estimation may be computed as

$$
\left(\begin{array}{c}
u \\
\tilde{u}
\end{array}\right)=\mathcal{G}^{\mathrm{T}} \mathcal{C}^{-1}\left(\begin{array}{c}
s \\
\tilde{s}
\end{array}\right)=\left(\begin{array}{c}
G^{\mathrm{T}} C^{-1}(I-P) s \\
\widetilde{G}^{\mathrm{T}} \widetilde{C}^{-1} \tilde{s}
\end{array}\right)
$$

where $\widetilde{G}$ and $\widetilde{C}$ are low-rank matrices. The second and final step in the evaluation, is to efficiently solve the system

$$
B \hat{\varphi}=\left(\begin{array}{c}
u \\
\tilde{u}
\end{array}\right) .
$$


To proceed with this computation, we decompose the matrix $B$ into blocks corresponding to layers:

$$
\begin{gathered}
B=\mathcal{M}-U U^{\mathrm{T}}+V V^{\mathrm{T}}, \\
\mathcal{M}=G^{\mathrm{T}} C^{-1} G+\mathcal{L}^{-1} .
\end{gathered}
$$

where $U$ and $V$ are low-rank matrices $(\operatorname{rank}(U)=2 H, \operatorname{rank}(V)=2 T)$ with block representation

$$
\begin{gathered}
U=\left(U_{l}\right)_{1 \leq l \leq L}, \quad U_{l}=\left(\left(G_{h l}\right)^{\mathrm{T}} \mathcal{Q}_{h}\right)_{1 \leq h \leq H}, \quad \mathcal{Q}_{h}=C_{h}^{-1} K_{h}\left(K_{h}^{\mathrm{T}} C_{h}^{-1} K_{h}\right)^{-1 / 2} \\
V=\left(V_{l}\right)_{1 \leq l \leq L}, \quad V_{l}=\left(\left(\widetilde{G}_{t l}\right)^{\mathrm{T}} \widetilde{C}_{t}^{-1 / 2}\right)_{1 \leq t \leq T} .
\end{gathered}
$$

The block decomposition of $B$ then reads

$$
B_{l l^{\prime}}=\mathcal{M}_{l l^{\prime}}-\sum_{h=1}^{H}\left(H_{h l}^{\varphi}\right)^{\mathrm{T}} \Gamma_{h}^{\mathrm{T}} C_{h}^{-1} P_{h} \Gamma_{h} H_{h l^{\prime}}^{\varphi}+\sum_{t=1}^{T}\left(\widetilde{H}_{t l}^{\varphi}\right)^{\mathrm{T}} \widetilde{\Gamma}_{t}^{\mathrm{T}} \widetilde{C}_{t}^{-1} \widetilde{\Gamma}_{t} \widetilde{H}_{t l^{\prime}}^{\varphi}
$$

\subsection{Turbulence Covariance Matrix Approximation}

Assuming that turbulence is stationary (i.e. the autocorrelation function depends only on the position difference between the pairs of positions involved), the atmospheric phase covariance matrix for layer $l, \mathcal{L}_{l}$, is a block Toeplitz with Toeplitz blocks (BTTB) matrix. Unfortunately, there is no simple formula for the inverse of BTTB matrices. Therefore, such matrices are often approximated by block circulant with circulant blocks (BCCB) matrices. This approximation is equivalent to a periodic extension of the phase screen. Consequently, on a finite grid, the BCCB approximation introduces a low-frequency error which will be neglegible provided the computational domain is large compared to the telescope diameter.

The BCCB approximation allows us to easily invert the covariance matrix. Indeed, BCCB matrices are diagonalized by the 2-D discrete Fourier transform (DFT), so we can write

$$
\mathcal{L}_{l} \simeq \mathcal{F}^{-1} \Lambda_{l} \mathcal{F}
$$

where $\mathcal{F}$ is the 2-D DFT matrix. A good approximation to $\mathcal{L}_{l}^{-1}$, which appears in the reconstructor matrix (3.1), is then

$$
\mathcal{L}_{l}^{-1} \simeq \mathcal{Z}_{l} \stackrel{\text { def }}{=} \mathcal{F}^{-1} \Lambda_{l}^{-1} \mathcal{F} .
$$

This matrix is also BCCB. The diagonal entries of $\Lambda$ come from the von Karman spatial power spectral density (PSD) of the atmospheric refractive index fluctuations $\delta n$, with the universal $-11 / 3$ power law:

$$
\Lambda_{l}(\kappa)=\left\langle|\delta n(\kappa)|^{2}\right\rangle=c_{l}^{2} /\left[|\kappa|^{2}+1 / L_{0}^{2}\right]^{11 / 6} .
$$

Since $\Lambda_{l}(\kappa)$ is positive, $\mathcal{Z}_{l}$ is also symmetric positive definite (SPD). Here $\kappa$ is the spatial frequency vector (in rads/meter), $L_{0}$ is the turbulence outer-scale, and $c_{l}$ is a constant defining the strength of layer $l$. For the case of a Kolmogorov turbulence spectrum, $L_{0}=\infty$.

$\mathcal{Z}_{l}$ is a non-sparse full rank matrix. As suggested in Ref.9, this matrix can be approximated by $\mathcal{S}_{l}^{2}$, a scaled discrete Laplacian squared matrix (also known as biharmonic operator) with periodic boundary. Indeed, the discrete Laplacian $\mathcal{S}$ has the Fourier representation

$$
\begin{gathered}
\mathcal{S}=\mathcal{F}^{-1} \operatorname{diag}(\hat{v}) \mathcal{F}, \\
\hat{v}=4\left[\sin ^{2}\left(\pi \kappa_{x} \Delta x\right) / \Delta x^{2}+\sin ^{2}\left(\pi \kappa_{y} \Delta y\right) / \Delta y^{2}\right]_{\Delta x, \Delta y \rightarrow 0}^{\longrightarrow} 4 \pi^{2}\left(\kappa_{x}^{2}+\kappa_{y}^{2}\right)=4 \pi^{2}|\kappa|^{2} .
\end{gathered}
$$

The advantage of this approximation is that the discrete Laplacian $\mathcal{S}_{l}=c_{l}^{\prime} \mathcal{S}$ is sparse, enabling a sparse analog of the estimation matrix. If we adopt this approximation, the blocks of $\mathcal{M}$ in the estimation matrix are given by

$$
\mathcal{M}_{l l^{\prime}}=\sum_{h=1}^{H}\left(G_{h l}\right)^{\mathrm{T}} C_{h}^{-1} G_{h l^{\prime}}+\mathcal{S}_{l}^{2} \delta_{l l^{\prime}}, \quad 1 \leq l, l^{\prime} \leq L .
$$




\section{MINIMUM VARIANCE FITTING MATRIX}

The minimum variance fitting (MVF) matrix is formally given by

$$
\begin{gathered}
F=A^{-1} H^{a^{\mathrm{T}}} \mathcal{W} H^{\varphi}, \\
A=H^{a^{\mathrm{T}}} \mathcal{W} H^{a}+V V^{\mathrm{T}}+\alpha I,
\end{gathered}
$$

where $V$ is a low-rank matrix whose columns are independent vectors from the null space of $H^{a^{\mathrm{T}}} \mathcal{W} H^{a}$, including for each DM layer, piston and cancelling tip/tilt modes, which have no effect on the piston-removed residual wavefront variance. $\alpha$ is a positive regularization parameter that must be included for MCAO systems to account for the modes in the null space that are not known a priori. The first step of the fitting part of the reconstructor can be computed easily from the sparse plus low-rank decomposition of the weighting matrix $\mathcal{W}$. To solve the fitting system, we proceed along the same lines as for the estimation part, i.e we decompose $A$ into layered blocks:

$$
\begin{gathered}
A=\mathcal{M}-U U^{\mathrm{T}}+V V^{\mathrm{T}}, \\
\mathcal{M}=H^{a^{\mathrm{T}}} \mathcal{W}_{0} H^{a}+\alpha I, \\
U=H^{a^{\mathrm{T}}} \mathcal{W}_{1}=\left(U_{m \bar{n}}\right)_{\substack{1 \leq m \leq M \\
1 \leq \bar{n} \leq \bar{N}}}, \quad U_{m \bar{n}}=\left(H_{\bar{n} m}^{a}\right)^{\mathrm{T}} \omega_{\bar{n}}^{1 / 2} v_{1}, \\
V=\left(V_{m}\right)_{1 \leq m \leq M},
\end{gathered}
$$

where $\operatorname{rank}(V)=2$ (piston, tip-tilt), $\operatorname{rank}(U)=\bar{N}$. The layered decomposition of $A$ then reads

$$
\begin{gathered}
A_{l l^{\prime}}=\mathcal{M}_{l l^{\prime}}-\sum_{\bar{n}=1}^{\bar{N}} U_{l \bar{n}}\left(U_{l^{\prime} \bar{n}}\right)^{\mathrm{T}}+V_{l} V_{l^{\prime}}^{\mathrm{T}}=\mathcal{M}_{l l^{\prime}}-\sum_{\bar{n}=1}^{\bar{N}}\left[\left(H_{\bar{n} l}^{a}\right)^{\mathrm{T}} \omega_{\bar{n}} v_{1}\right]\left[v_{1}^{\mathrm{T}} H_{\bar{n} l^{\prime}}^{a}\right]+V_{l} V_{l^{\prime}}^{\mathrm{T}} \\
\mathcal{M}_{l l^{\prime}}=\sum_{\bar{n}=1}^{\bar{N}}\left(H_{\bar{n} l}^{a}\right)^{\mathrm{T}} \omega_{\bar{n}} W_{0} H_{\bar{n} l^{\prime}}^{a}+\alpha I_{l} \delta_{l l^{\prime}} .
\end{gathered}
$$

\section{MG LAYER-ORIENTED PCG}

From the above discussion, both MVE and MVF matrix systems require the solution of symmetric positive definite (SPD) block matrix systems of generic form

$$
A x=b,
$$

where $x$ is a concatenated vector whose support is either the set of atmospheric or DM layers. In both cases, the matrix $A$ can be represented in the form :

$$
\begin{gathered}
A=\mathcal{M}-U U^{\mathrm{T}}+V V^{\mathrm{T}}=\mathcal{M}-\mathcal{U} \mathcal{V}^{\mathrm{T}}, \\
\mathcal{U}=(U V), \quad \mathcal{V}=(U-V) .
\end{gathered}
$$

where $U$ and $V$ are low-rank full matrices. In order to solve (5.1), we use the Sherman-Morrison-Woodbury formula to invert low-rank perturbations to a matrix. The formula (also known as matrix inversion lemma) reads:

$$
\left(\mathcal{M}-\mathcal{U} \mathcal{V}^{\mathrm{T}}\right)^{-1}=\mathcal{M}^{-1}+\mathcal{M}^{-1} \mathcal{U}\left(I-\mathcal{V}^{\mathrm{T}} \mathcal{M}^{-1} \mathcal{U}\right)^{-1}\left(\mathcal{M}^{-1} \mathcal{V}\right)^{\mathrm{T}}
$$

Note that $\operatorname{rank}\left(I-\mathcal{V}^{\mathrm{T}} \mathcal{M}^{-1} \mathcal{U}\right)=\operatorname{rank}(U)+\operatorname{rank}(V)$. The problem is thus reduced to solving systems involving $\mathcal{M}$. For natural guide star (NGS) MCAO systems, $U$ and $V$ are null matrices for the estimation step. A simplified (non-block) version for the single-layer case has already been solved efficiently at a $\operatorname{cost}$ of $\mathcal{O}(n \log n)$ using a multigrid preconditioned conjugate gradient method. ${ }^{10}$ We propose to develop block extensions for the case of multiple layers.

The conjugate gradient (CG) method $^{18}$ is an iterative scheme for solving SPD linear systems (5.1). Starting with $x_{0}=0$, CG generates polynomial approximates $x_{\nu}=P_{\nu}(A) b$ to $x^{*}=A^{-1} b$. The polynomials $P_{\nu}$ are optimal in the sense that they minimize $\left\|e_{\nu}\right\|_{A}^{2}$ where $e_{\nu}=x_{\nu}-x^{*}$ and $\left\|e_{\nu}\right\|_{A}^{2}=e_{\nu}^{\mathrm{T}} A e_{\nu}$. If $A$ has $q$ distinct eigenvalues, $\mathrm{CG}$ is guaranteed to converge to the exact solution $x^{*}$ in at most $q$ iterations, and it is "matrix 
free" in the sense that it does not require explicit storage of the matrix $A$. Each iteration of CG requires one vector-matrix multiply $z=A v$. In $\mathrm{AO}$ applications, $A$ is very poorly conditioned, and this leads to very slow convergence of CG when applied directly to (5.1). Therefore, the required number of iterations may be unacceptably high. Preconditioning ${ }^{18}$ refers to a technique to speed up the convergence of iterative methods. A preconditioner $A^{\prime}$ is an SPD approximation of $A$ such that $\operatorname{cond}\left(A^{\prime-1} A\right) \ll \operatorname{cond}(A)$, i.e. $A^{\prime^{-1}} A$ has a more desirable eigenvalue structure (clustering of the eigenvalues and/or low condition number) leading to rapid convergence of CG. The preconditioned conjugate gradient (PCG) algorithm solves the equivalent system

$$
A^{\prime-1} A x=A^{\prime-1} b .
$$

Application of the preconditioner requires the computation of vector-matrix multiplies of the form $u=A^{\prime^{-1}} v$. The dominant costs of our PCG algorithm are the application of the preconditioner and vector-matrix multiplies involving coefficient matrix $A .{ }^{18}$ We propose a multigrid algorithm to implement this preconditioning step.

\subsection{The Multigrid Preconditioner}

Multigrid (MG) methods ${ }^{19}$ are extremely effective for solving strongly elliptic partial differential equations (PDE's) like the Laplace or biharmonic equations. The estimation matrix (3.11) falls into this class, and MG has been demonstrated to be very effective to solve (5.1) for ExAO systems. ${ }^{10}$ MG is a multiresolution method. The key idea of a 2-grid scheme is to apply an iterative method (smoother) with fast convergence for high frequencies, project the (low-frequency) error onto a coarser grid (restriction), solve the system on the coarse grid, project back the coarse grid solution error onto the fine grid (interpolation), update the solution on the fine grid and reapply the smoother to get rid of the new high-frequencies introduced. Multigrid methods are a recursive application of the 2 -grid idea. To solve $A^{\prime} u=v, \mathrm{MG}$ requires the following components:

(i) A nested sequence of grids where $e_{h}=A_{h}^{-1} r_{h}$ is defined.

(ii) Restriction and interpolation operators (transpose of each other to preserve symmetry for CG).

(iii) A smoother: a stationary iterative method (characterized by an iteration matrix) which rapidly damps out high frequencies of the residual at each grid level.

A typical MG V-cycle can be found in Ref.19.

\subsection{The layer-oriented Symmetric Gauss-Seidel Smoother}

The recent "layer-oriented" control algorithms for $\mathrm{MCAO}^{12-15}$ suggest to use a layer-oriented preconditioner. The layer-oriented approach uses WFS's conjugated to the DM's instead of to the entrance pupil, and an optical scheme to combine the signals from all guide stars. This has the effect of diminishing the cross-coupling of the reconstructor, such that each WFS can control its corresponding DM directly. It is argued that such systems may be less complex and less demanding in terms of computation.

In our computational approach, the first step in the estimation matrix $E$ (i.e $\mathcal{G}^{\mathrm{T}} \mathcal{C}^{-1}$ ) numerically combines the measurements from multiple WFS's in the same way as Ragazzoni's optical scheme with pyramid wavefront sensors. Therefore, we propose a layer-oriented (block) symmetric Gauss-Seidel (SGS) iteration as a smoother for MG. The iteration is based on the following partitioning of $A$ :

$$
A=A_{\mathrm{D}}-A_{\mathrm{L}}-A_{\mathrm{U}},
$$

where $A_{\mathrm{D}}, A_{\mathrm{L}}$ and $A_{\mathrm{U}}$ are block matrices assembled from the diagonal, lower and upper blocks of $A$ respectively. The SGS iteration reads

$$
\begin{gathered}
x^{(2 k-1)}=\left(A_{\mathrm{D}}+A_{\mathrm{L}}\right)^{-1} y^{(2 k-2)}, \quad x^{(2 k)}=\left(A_{\mathrm{D}}+A_{\mathrm{U}}\right)^{-1} y^{(2 k-1)}, \\
y^{(2 k-2)} \stackrel{\text { def }}{=} b-A_{\mathrm{U}} x^{(2 k-2)}, \quad y^{(2 k-1)} \stackrel{\text { def }}{=} b-A_{\mathrm{L}} x^{(2 k-1)} .
\end{gathered}
$$


For layer $l(1 \leq l \leq L)$, these read

$$
\begin{gathered}
y_{L}^{(2 k-2)}=b_{L}^{(2 k-2)}, \quad y_{l}^{(2 k-2)}=b_{l}-\sum_{l^{\prime}=l+1}^{L} A_{l l^{\prime}} x_{l^{\prime}}^{(2 k-2)}, \\
y_{1}^{(2 k-1)}=b_{1}^{(2 k-1)}, \quad y_{l}^{(2 k-1)}=b_{l}-\sum_{l^{\prime}=1}^{l-1} A_{l l^{\prime}} x_{l^{\prime}}^{(2 k-1)} .
\end{gathered}
$$

Note that the iteration preserves cross-coupling between layers and can be implemented using back-substitutions:

$$
\begin{aligned}
x_{l}^{(2 k-1)} & =A_{l l}^{-1}\left[y_{l}^{(2 k-2)}-\sum_{l^{\prime}=1}^{l-1} A_{l l^{\prime}} x_{l^{\prime}}^{(2 k-1)}\right], \\
x_{l}^{(2 k)} & =A_{l l}^{-1}\left[y_{l}^{(2 k-1)}-\sum_{l^{\prime}=l+1}^{L} A_{l l^{\prime}} x_{l^{\prime}}^{(2 k)}\right],
\end{aligned}
$$

which requires inversion of only diagonal blocks

$$
A_{l l}=\mathcal{M}_{l l}-U_{l} U_{l}^{\mathrm{T}}+V_{l} V_{l}^{\mathrm{T}}, \quad 1 \leq l \leq L .
$$

This is done again using the matrix inversion lemma (5.3) implemented with Cholesky factorizations of the diagonal blocks $\mathcal{M}_{l l}$ (complete or incomplete) with much less fill-in than factorization of the full matrix $\mathcal{M}$ since off-diagonal blocks have been eliminated. The computational cost and storage of the Cholesky factorizations scale as the number of layers times $3 / 2$ power of the number of grid points per layer.

\section{FITTING STEP}

The basic approach for the fitting step is incomplete Cholesky-preconditioned CG. ${ }^{18}$ The incomplete Cholesky factorization is applied to the sparse component $\mathcal{M}$ of the fitting matrix, producing an approximate factorization which has the same sparsity pattern as the upper triangular part of $\mathcal{M}$. Both storage and cost per CG iteration for this step scale as $\mathcal{O}(n)$. Fast convergence (see Fig.5) implies overall cost of fitting is $\mathcal{O}(n)$.

\section{NUMERICAL SIMULATION RESULTS}

We have arrived at a general framework for minimum variance reconstructors, with a variety of options for special cases. The basic approach for the estimation step is the multigrid-preconditioned CG with a layer-oriented (block) SGS smoothing. Using a sparse representation for the turbulence inverse covariance matrix, diagonal blocks of the SGS smoother can be inverted using a symmetric approximate minimum degree (SYMAMD) permutation $^{20}$ prior to Cholesky factorization to avoid fill-in. For the fitting step, the basic approach is incomplete Cholesky-preconditioned CG. ${ }^{18}$ Low rank perturbations for LGS and tip-tilt NGS measurements are easily accounted for using the matrix inversion lemma. Exact representation of the turbulence statistics requires an inner iterative solver like multigrid to invert the diagonal blocks $\mathcal{M}_{j j}$, which greatly increases the overall computational cost.

Figure 4 shows typical convergence results for the estimation step for telescope diameters equal to $32 \mathrm{~m}, 16 \mathrm{~m}$ and $8 \mathrm{~m}$. We consider the following 6-layer atmospheric profile obtained using generalized SCIDAR measurements at Cerro Pachon, Chile:

\begin{tabular}{|l|l|l|l|l|l|l|}
\hline Layer & 1 & 2 & 3 & 4 & 5 & 6 \\
\hline Altitude $(\mathrm{m})$ & 0 & 2580 & 5160 & 7730 & 12890 & 15460 \\
\hline Relative weight & 0.652 & 0.172 & 0.055 & 0.025 & 0.074 & 0.022 \\
\hline
\end{tabular}

The MCAO system under consideration has $N=5$ NGS's at the edges and center of a FoV of diameter 100 arcsec. We are considering an array of $5 \times 5$ observation directions partioning the FoV. The weights $\omega_{\bar{n}}$ of the weighting matrix $\mathcal{W}$ in (2.8) have been chosen each equal to $1 / \bar{N}=1 / 25$. Our WFS sampling is at half phase screen resolution. The estimation error norm averaged over the FoV has been computed as

$$
\begin{gathered}
\left\|\epsilon_{\mathrm{est}}\right\|^{2} \stackrel{\text { def }}{=} \epsilon_{\mathrm{est}}^{\mathrm{T}} \mathcal{W} \epsilon_{\mathrm{est}} /\left(H^{\varphi} \varphi\right)^{\mathrm{T}} \mathcal{W}\left(H^{\varphi} \varphi\right) \\
\epsilon_{\mathrm{est}}=H^{\varphi}(\hat{\varphi}-\varphi)
\end{gathered}
$$


Comparison with direct matrix inversion has been made using the following error metric:

$$
\begin{gathered}
\left\|\epsilon_{\mathrm{ex}}\right\|^{2} \stackrel{\text { def }}{=} \epsilon_{\mathrm{ex}}^{\mathrm{T}} \mathcal{W} \epsilon_{\mathrm{ex}} /\left(H^{\varphi} \hat{\varphi}_{\mathrm{ex}}\right)^{\mathrm{T}} \mathcal{W}\left(H^{\varphi} \hat{\varphi}_{\mathrm{ex}}\right) \\
\epsilon_{\mathrm{ex}}=H^{\varphi}\left(\hat{\varphi}-\hat{\varphi}_{\mathrm{ex}}\right)
\end{gathered}
$$

Finally, the rms estimation error averaged over the Fov displayed in the lower right panel of Figure 4 has been computed as

$$
\xi_{\text {est }}=\left(\sum_{\bar{n}=1}^{\bar{N}} \omega_{\bar{n}}\left(H^{\varphi} \hat{\varphi}-H^{\varphi} \varphi\right)_{\bar{n}}^{2} / \sum_{\bar{n}=1}^{\bar{N}} \omega_{\bar{n}}\left\|\left(H^{\varphi} \varphi\right)_{\bar{n}}\right\|^{2}\right)^{1 / 2}
$$

where component-wise operation has to be understood for the square appearing in the numerator and the overall square root. We used $1 \mathrm{~V}$-cycle/CG iteration and $1 \mathrm{SGS}$ iteration/grid level. $r_{0}$ is equal to $25 \mathrm{~cm}$ and the phase screens resolution is $\Delta x=r_{0}$. We used phase screens twice the size of the telescope diameter $D$, i.e. $256 \times 256$, $128 \times 128$, and $64 \times 64$ for the $D=32 \mathrm{~m}, 16 \mathrm{~m}$, and $8 \mathrm{~m}$ cases respectively. The table below summarizes the number of grid points/phase screen, subapertures/WFS and actuators/DM for the different cases considered:

\begin{tabular}{|l|l|l|l|}
\hline $\mathrm{D}(\mathrm{m})$ & \#grid points/phase screen & \#subapertures/WFS & \#actuators/DM \\
\hline 8 & $64 \times 64$ & 220 & 276 \\
\hline 16 & $128 \times 128$ & 832 & 932 \\
\hline 32 & $256 \times 256$ & 3208 & 3408 \\
\hline
\end{tabular}

Figure 5 compares convergence results for the fitting step as the number of DM's varies from 1 to 3 . In the 3 -DM configuration, DM's are conjugated to $0 \mathrm{~m}, 5160 \mathrm{~m}$, and $10320 \mathrm{~m}$; in the 2-DM case, conjugation altitudes are $0 \mathrm{~m}$ and $2580 \mathrm{~m}$; in the single-DM case, conjugation altitude is $0 \mathrm{~m}$. All DM's are at half the phase screens resolution. The lower right pannel of Figure 5 diplays the residual error after $2 \mathrm{CG}$ fitting iterations for the 3 -DM configuration as a function of CG estimation iterations.
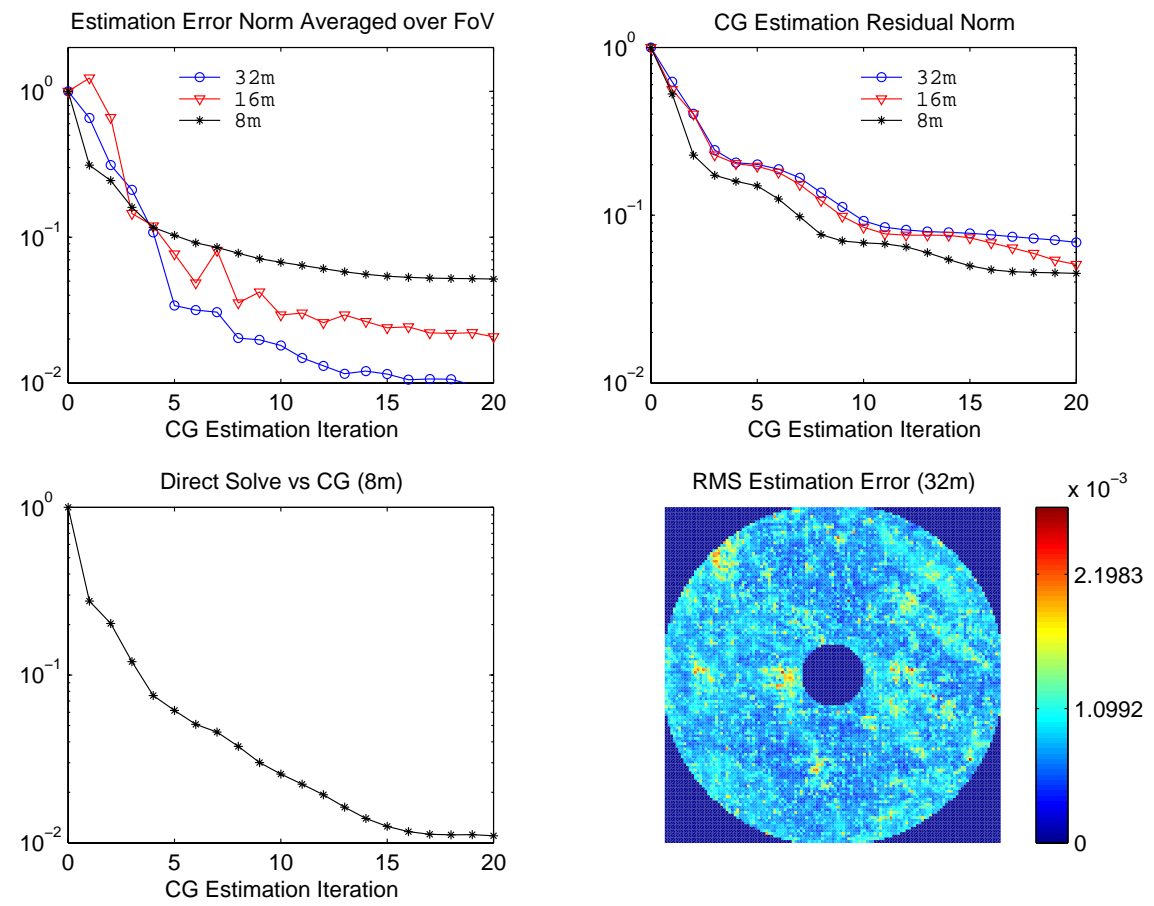

Figure 4. Minimum variance estimation for the Cerro-Pachon 6-layer profile, telescopes diameters equal to $32 \mathrm{~m}$, $16 \mathrm{~m}$ and $8 \mathrm{~m}$. 5 WFSs' using 5 NGS's at the edges and center of a FoV of diameter 100 arcsec, 1 V-cycle/CG iteration, 1 SGS iteration/grid level, $\mathrm{SNR}=20, r_{0}=25 \mathrm{~cm}, \Delta x=r_{0}$. 

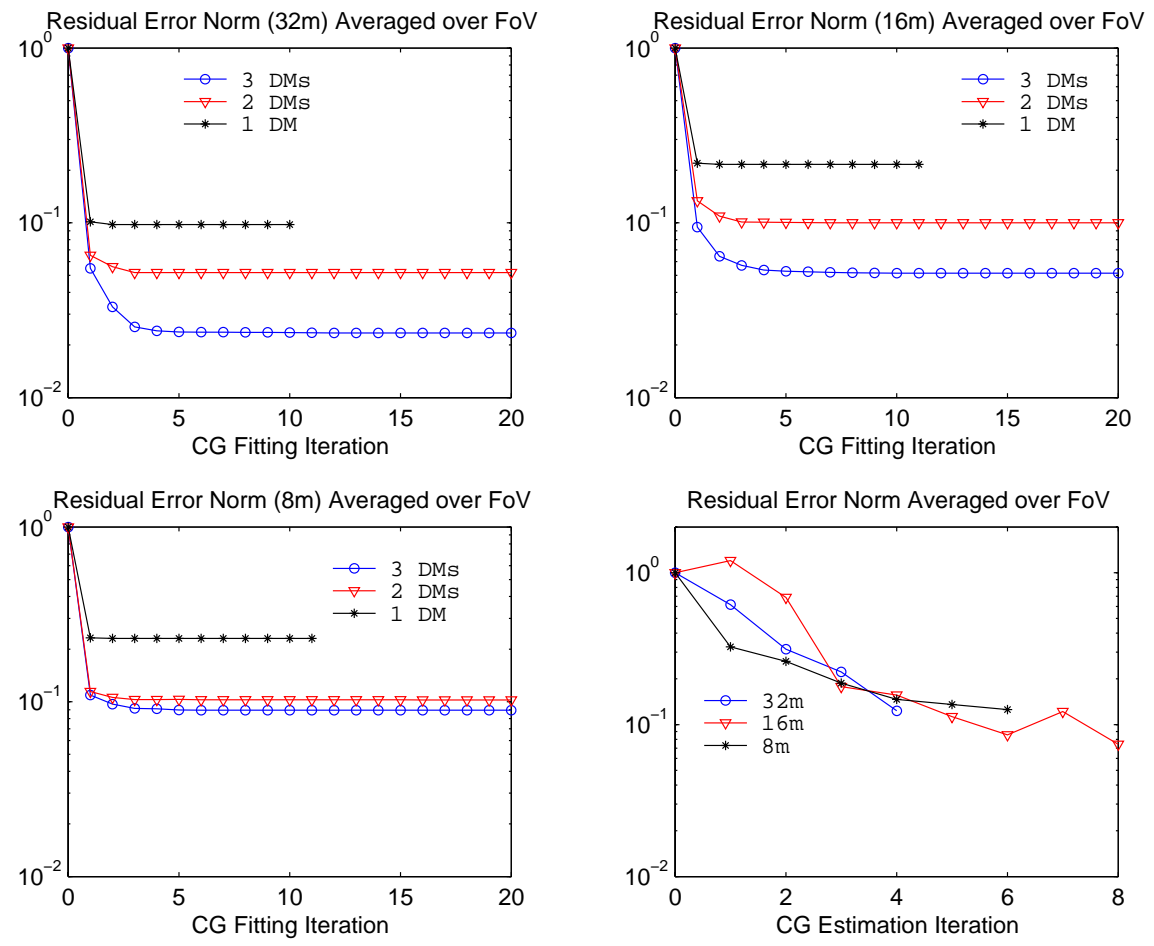

Figure 5. Minimum variance fitting for the same system as in Fig. 4 with 3 different DM configurations. 20 CG estimation iterations were taken before starting the fitting step. Averaging over an array of $5 \times 5$ observation directions partitioning a FoV of diameter 100 arcsec. Incomplete Cholesky preconditioning, regularization parameter $\alpha=10^{-5}$, SNR $=20$, $r_{0}=25 \mathrm{~cm}, \Delta x=r_{0}$.

\section{CONCLUSIONS}

We have developed efficient layer-oriented multigrid wavefront reconstruction algorithms for open-loop multiconjugate adaptive optics systems on $32 \mathrm{~m}, 16 \mathrm{~m}$ and $8 \mathrm{~m}$ telescopes. For the estimation step, the basic approach is the conjugate gradient method with a multigrid preconditioner to speed up convergence, and a layer-oriented (block) symmetric Gauss-Seidel smoother. Using a sparse representation for the turbulence inverse covariance matrix, diagonal blocks of the SGS smoother can be inverted using a symmetric approximate minimum degree (SYMAMD) permutation prior to Cholesky factorization to avoid fill-in. For the fitting step, the basic approach is incomplete Cholesky-preconditioned CG. Low rank perturbations for LGS and tip-tilt NGS measurements are easily accounted for using the matrix inversion lemma.

\section{ACKNOWLEDGMENTS}

We acknowledge the support of the US Air Force Office of Scientific Research and the Gemini Observatory, which is operated by the Association of Universities for Research in Astronomy, Inc., under a cooperative agreement with the NSF on behalf of the Gemini Partnership: the National Science Foundation (United States), the Particle Physics and Astronomy Research Council (United Kingdom), the National Research Council (Canada), CONICYT (Chile), the Australian Research Council (Australia), CNPq (Brazil), and CONICET (Argentina).

\section{REFERENCES}

1. M. C. Roggemann and B. Welsh, Imaging through turbulence, CRC Press, 1996.

2. J. W. Hardy, Adaptive Optics for Astronomical Telescopes, Oxford University Press, 1998.

3. F. J. Roddier, Adaptive Optics for Astronomy, Cambridge University Press, 1999. 
4. J. M. Beckers, "Increasing the size of the isoplanatic patch with multi-conjugate adaptive optics," in Proc. European Southern Observatory Conference and Workshop on Very Large Telescopes and their Instrumentation, M. H. Ulrich, ed., 30, 693-703, 1998.

5. D. C. Johnston and B. M. Welsh, "Analysis of multi-conjugate adaptive optics," J. Opt. Soc. Am. A11, 394-408, 1994.

6. B. L. Ellerbroek, "First order performance evaluation of adaptive-optics systems for atmospheric turbulence compensation in extended field-of-view astronomical telescopes," J. Opt. Soc. Am. A11, 783-805, 1994.

7. T. Fusco, J. M. Conan, G. Rousset, L. M. Mugnier, and V. Michau, "Optimal wave-front reconstruction strategies for multi-conjugate adaptive optics," J. Opt. Soc. Am. A18, 2527-2538, 2001.

8. B. L. Ellerbroek, "Methods for correcting tilt anisoplanatism in laser-guide-star based multi-conjugate adaptive optics," J. Opt. Soc. Am. A18, 2539-2547, 2001.

9. B. L. Ellerbroek, "Efficient computation of minimum variance wavefront reconstructors using sparse matrix techniques," J. Opt. Soc. Am. A19, September, 2002.

10. L. Gilles, C .R. Vogel and B. L. Ellerbroek, "A multigrid preconditioned conjugate gradient method for large scale wavefront reconstruction," J. Opt. Soc. Am. A19, September, 2002.

11. L. A. Poyneer, D. T. Gavel and J. M. Brase, "Fast wavefront reconstruction in large adaptive optics systems using the Fourier transform," manuscript available from Lisa Poyneer, Lawrence Livermore National Laboratory, 2002.

12. R. Ragazzoni, J. Farinato and E. Marchetti, "Adaptive optics for $100 \mathrm{~m}$ class telescopes: new challenges require new solutions," in Beyond Conventional Adaptive Optics, http://lenin.pd.astro.it/venice2001/proceedings/, Proc. ESO, 2001.

13. R. Ragazzoni, E. Diolaiti, J. Farinato, E. Fedrigo, E. Marchetti, M. Tordi and D. Kirkman, "Multiple Field of View Layer Oriented," in Beyond Conventional Adaptive Optics, http://lenin.pd.astro.it/venice2001/proceedings/, Proc. ESO, 2001.

14. M. Tordi, R. Ragazzoni and E. Diolaiti, "Simulation of a Layer Oriented MCAO system," in Beyond Conventional Adaptive Optics, http://lenin.pd.astro.it/venice2001/proceedings/, Proc. ESO, 2001.

15. A. A. Tokovinin, "Maximum separation between guide stars in atmospheric tomography," in Beyond Conventional Adaptive Optics, http://lenin.pd.astro.it/venice2001/proceedings/, Proc. ESO, 2001.

16. B. L. Ellerbroek, "Scaling multi-conjugate adaptive optics performance estimates to extremely large telescopes," Proc. SPIE 4007, pp. 1088-1099, 2000.

17. O. Axelsson and V. A. Barker, Finite Element Solution of Boundary Value Problems: Theory and Computation, SIAM, Philadelphia, 2001.

18. Y. Saad, Iterative Methods for Sparse Linear Systems, PWS Publishing Company, Boston, 1996.

19. U. Trottenberg, C. W. Oosterlee and A. Schüller, Multigrid, Academic Press, London, 2001.

20. T. A. Davis, J. R. Gilbert, S. I. Larimore and E. G. Ng, "A column approximate minimum degree ordering algorithm," submitted to ACM Trans. on Mathematical Software. Available at http://www.cise.ufl.edu/research/sparse/colamd/ 